\title{
SUPREMUM NORM DIFFERENTIABILITY
}

\author{
I.E. LEONARD and K.F. TAYLOR \\ Department of Mathematics, University of Saskatchewan, \\ Saskatoon, Saskatchewan, Canada S7N OWO \\ (Received June 1, 1982)
}

ABSTRACT. The points of Gateaux and Frechet differentiability of the norm in $C(T, E)$ are obtained, where $T$ is a locally compact Hausdorff space and $E$ is a real Banach space. Applications of these results are given to the space $\ell_{\infty}(E)$ of all bounded sequences in $E$, and to the space $B\left(\ell_{1}, E\right)$ of all bounded linear operators from $\ell_{1}$ into $E$.

KEY WORDS AND PHRASES. Banach spaces, continuous functions, vector-valued functions, supremum norm, smooth points.

1980 MATHEMATICS SUBJECT CLASSIFICATION CODE. Primary: 46B20, $46 E 40$. Secondary: 46A32, 46A45.

1. INTRODUCTION.

In [1], Banach proved that if $T$ is a compact metric space and $C(T)$ is the Banach space of all continuous real valued functions on $T$, with the supremum norm, then

$$
\lim _{\lambda \rightarrow 0} \frac{\|f+\lambda g\|-\|f\|}{\lambda}
$$

exists for all $g \in C(T)$ if and only if there exists a $t_{0} \varepsilon T$ such that $\left|f\left(t_{0}\right)\right|>|f(t)|$ for all $t \in T, \quad t \neq t_{0}$. 
This theorem, however, is no longer true if $T$ is a locally compact, noncompact, Hausdorff space; as can easily be seen by considering the Banach space $\ell_{\infty}$ of all bounded real valued sequences with the supremum norm.

In fact, if $\mathbb{N}$ is the set of positive integers equipped with the discrete topology, then $\ell_{\infty}=C(\mathbb{N})$, the space of all bounded continuous functions on $\mathbb{N}$. If we let $x=\left\{x_{n}\right\}_{n \geq 1} \varepsilon \ell_{\infty}$, where $x_{1}=1$ and $x_{n}=\frac{n-1}{n}$ for $n>1$, then $x$ peaks at $n=1$, but because of the behaviour of $x$ at infinity and the existence of Banach limits, it is possible to find two distinct support functionals to the ball in $\ell_{\infty}$ at $x$, so that $x$ is not a smooth point.

In this note, we characterize the points of Gateaux and Fréchet differentiability of the norm function in $C(T, E)$, the space of all bounded continuous E-valued functions on the locally compact Hausdorff space $T$, where $E$ is a real Banach space.

Two applications of these results are given. The first is to the space $\ell_{\infty}(E)$ of all bounded sequences in $E$, and the second to the space $B\left(\ell_{j}, E\right)$ of all bounded linear operators from $\ell_{1}$ into $E$.

\section{DEFINITIONS AND NOTATION.}

In the following, $E$ denotes a real Banach space and $E^{\star}$ denotes the dual of $E$. The unit ball of $E$ is $B_{E}=\{x \in E \mid\|x\| \leq 1\}$ and its boundary $S_{E}=\left\{x \in E \mid\|x\|_{1}=1\right\}$ is the unit sphere of $E$.

A Banach space $E$ is said to be smooth at $x \in E \sim\{0\}$ if and only if there exists a unique hyperplane of support to $B_{E}$ at $\frac{x}{\|x\|}$; that is, there exists only one continuous linear functional $\phi \in E^{*}$ with $\|\phi\|=1$ such that $\phi(x)=\|x\|$. Such a linear functional $\phi \varepsilon E^{\star}$ is called the support functional to $B_{E}$ at $\frac{x}{\|x\|}$, and $\phi^{-1}(\{1\})$ is called the hyperplane of support to $B_{E}$ at $\frac{x}{\|x\|}$. A Banach space $E$ is said to be a smooth Banach space if it is smooth at every $x \in S_{E}$.

The norm function $\|\cdot\|: E \rightarrow \mathbb{R}^{+}$is said to be Gateaux differentiable at $x \in E \sim\{0\}$ if and only if there exists a functional $\phi \varepsilon E^{*}$ with

$$
\lim _{\lambda \rightarrow 0}\left|\frac{\|x+\lambda h\|-\|x\| i}{\lambda}-\phi(h)\right|=0,
$$


for every $h \in E$. The functional $\phi$ is called the Gateaux derivative of the norm at $x \in E$. The norm function $\|\cdot\|: E \rightarrow \mathbb{R}^{+}$is said to be Fréchet differentiable at $X \in E \sim\{0\}$ if and only if there exists a functional $\phi \varepsilon E^{*}$ such that

$$
\lim _{\|h\| \rightarrow 0} \frac{\perp\|x+h i \mid-\| x \|-\phi(h)\rfloor}{\|h\|}=0,
$$

that is, the limit in $(*)$ exists uniformly for $h \in B_{E}$.

It is well known, Mazur [8], that $E$ is smooth at $x \in E \sim\{0\}$ if and only if $\lim _{\lambda \rightarrow 0} \frac{\|x+\lambda h\|-\|x\|}{\lambda}$ exists for all $h \in E$, if and only if the norm function $\|\cdot\|^{\prime}: E \rightarrow R^{+}$is Gateaux differentiable at $x$.

3. SMOOTH POINTS IN $C(T, E)$.

If $T$ is a topological space and $E$ is a real Banach space, then $C(T, E)$ denotes the Banach space of all bounded continuous $E$-valued functions on $T$, with the supremum norm; that is,

$$
C(T, E)=\{f: T \rightarrow E \mid f \text { is bounded and continuous }\},
$$

and $\|f\|=\sup \{\|f(t)\|: t \varepsilon T\}$, for $f \in C(T, E)$.

As mentioned earlier, Banach [1] proved that if $T$ is a compact metric space, then $C(T)=C(T, \mathbb{R})$ is smooth at $f \neq 0$ if and only if $f$ is a peaking function, that is, there exists a point $t_{0} \varepsilon T$ such that $\|f\|=\left|f\left(t_{0}\right)\right|>|f(t)|$ for all $t \in T, \quad t \neq t_{0}$.

Kondagunta [6], and Cox and Nadler [3], have characterized the points of Gateaux and Fréchet differentiability of the norm in $C(T, E)$ when $T$ is compact Hausdorff. Cox and Nadler, in the same paper, give a characterization of the points of Fréchet differentiability of the norm in $C(T, \mathbb{R})$ when $T$ is locally compact Hausdorff.

In this section, we generalize these results to the space $C(T, E)$ when $T$ is locally compact Hausdorff. The techniques used by Cox and Nadler will not work in this case, since, in general, the range of $f \in C(T, E)$ is no longer relatively compact and hence no extension to an $\hat{f} \in C(B T, E)$ is possible. However, a slight modification of the argument given in $k \Delta$ the [7], (page 352), for the corresponding result in $\ell_{\infty}$, does work. 
As usual, the results on smoothness are the more difficult, and the results on Fréchet differentiability follow as a corollary.

THEOREM 3.1. Let $T$ be a locally compact Hausdorff space and $E$ a Banach space. A point $f \in C(T, E), \quad f \neq 0$, is a smooth point of $C(T, E)$ if and only if

(i) there exists a $t_{0} \varepsilon T$ such that $\left\|f\left(t_{0}\right)\right\|>\|f(t)\|$ for all $t \in T$, $t \neq t_{0}$,

(ii) there exists a compact neighborhood $k$ of $t_{0}$ such that

$$
\sup _{t \in T \sim K}\|f(t)\|<\|f\|
$$

(iii) $f\left(t_{0}\right)$ is a smooth point of $E$.

PROOF.

A. Assume first that the norm $\|\|:. C(T, E) \rightarrow \mathbb{R}^{+}$is Gateaux differentiable at $f \in C(T, E)$, where we may (and do) assume that $\|f\|=1$.

(I) We show first that if the mapping $\|f()\|:. T \rightarrow \mathbb{R}^{+}$achieves its maximum at $t_{0} \varepsilon T$, then $t_{0}$ is unique. Suppose there exist $t_{0}, t_{1} \varepsilon T, t_{0} \neq t_{1}$, such that $\left\|f\left(t_{0}\right)\right\|=\left\|f\left(t_{1}\right)\right\|=1$. For $t \varepsilon T$ and $\phi \varepsilon E^{\star}$, let $\delta_{\phi, t} \varepsilon C(T, E)^{\star}$ denote the evaluation functional given by $\delta_{\phi, t}(g)=\phi(g(t))$ for $g \varepsilon C(T, E)$; then $\left\|\delta_{\phi, t}\right\|=1$ for all $t \varepsilon T, \phi \varepsilon S_{E^{*}}$. Using the Hahn-Banach theorem, choose $\phi_{0}, \phi_{1} \varepsilon E^{\star}$ with $\left\|\phi_{0}\right\|=\left\|\phi_{1}\right\|=1$ such that $\phi_{0}\left(f\left(t_{0}\right)\right)=\phi_{1}\left(f\left(t_{1}\right)=1\right.$. Then $\delta_{\phi_{0}, t_{0}}$ and $\delta_{\phi_{1}, t_{1}}$ are distinct support functionals to the ball in $C(T, E)$ at $f$, which contradicts the fact that $f$ is a smooth point in $C(T, E)$.

(II) We show next that given any compact set $K \subseteq T$, either $\sup _{t \varepsilon T \sim K^{\circ}}\|f(t)\|<1$, or there exists a $t_{0} \varepsilon T \sim K^{\circ}$ such that $\left\|f\left(t_{0}\right)\right\|=1$.

To the contrary, suppose there exists a compact set $K \subseteq T$, such that $\sup _{t \varepsilon T \sim K^{\circ}}\|f(t)\|=1$ and $\|f(t)\|<1$, for all $t \in T \sim K^{\circ}$. Let $F_{0}(t)=\|f(t)\|$, for all $t \in T$. Then $F_{0}$ is a bounded continuous function on $T$ and thus has an extension, $F$, to $B T$, the Stone-Čech compactification of $T$. Since $\sup _{t \varepsilon T \sim K^{\circ}}\|f(t)\|=1$, we have $A=F^{-1}(1) \cap(B T \sim T) \neq \phi . \quad$ Also

$$
A=\bigcap_{n=1}^{\infty}\left\{t \varepsilon T \sim K: F(t)>1-\frac{1}{n}\right\}
$$


and thus $A$ is a $G_{\delta}$ set in $B T$. By Čech [2], singletons in $B T \sim T$ are not $G_{\delta}$ sets, so $A$ contains at least two distinct points $p$ and $q$.

Let $\left\{p_{\mu}\right\}$ and $\left\{q_{\nu}\right\}$ be disjoint nets contained in $T$ such that $p_{\mu} \rightarrow p$ and $q_{\nu} \rightarrow q$ in $B T$. For each $\mu$, let $\phi_{\mu} \in E^{*}$, with $\left\|\phi_{\mu}\right\|=1$ and $\phi_{\mu}\left(f\left(p_{\mu}\right)\right)=F\left(p_{\mu}\right)$. Also, for each $\nu$, choose $\psi_{\nu} \varepsilon E^{*}$, with $\left\|\psi_{\nu}\right\|=1$ and $\psi_{\nu}\left(f\left(q_{\nu}\right)\right)=F\left(q_{\nu}\right)$. Let $\Phi_{\mu}=\delta_{\phi_{\mu}, p_{\mu}}$ and $\psi_{\nu}=\delta_{\psi_{\nu}, q_{\nu}}$, for each $\mu$ and $\nu$. Then $\Phi_{\mu}$ and $\Psi_{\nu} \in C(T, E) *$ and $\left\|\Phi_{\mu}\right\|=\left\|\Psi_{\nu}\right\|=1$, for each $\mu$ and $\nu$.

Since the ball in $C(T, E)$ * is $w^{\star}$-compact, there exist $\Phi, \Psi \varepsilon C(T, E)^{\star}$, with $\|\Phi\| \leq 1$ and $\|\Psi\| \leq 1$, such that $\Phi$ is a $w^{*}$-accumulation point of the net $\left\{\Phi_{\mu}\right\}$ and $\Psi$ is a $w^{*}$-accumulation point of the net $\left\{\Psi_{\nu}\right\}$. By construction, $\Phi(f)=\Psi(f)=1$ and, thus, $\Phi$ and $\Psi$ are support functionals to the ball in $C(T, E)$ at $f$. Since $f$ is assumed to be a smooth point in $C(T, E)$, it must be that $\Phi=\psi$. We will show that this is impossible. Let $P=\left\{p_{\mu}\right\} U\{p\}$ and $Q=\left\{q_{\nu}\right\} U\{q\}$. Then $P$ and $Q$ are disjoint closed subsets of $B T$, which is $a$ compact Hausdorff space and therefore normal. Let $h_{1}, h_{2} \varepsilon C(B T)$ with $0 \leq h_{1}, h_{2} \leq 1, h_{1}+h_{2}=1$ and $h_{1}(P)=h_{2}(Q)=0$. Use $h_{i}$ for the restriction of $h_{j}$ to $T$, as well.

Clearly, if $g \in C(T, E)$, then $h_{1} g \varepsilon \operatorname{ker} \Phi$ and $h_{2} g \varepsilon \operatorname{ker} \psi$. Since $\Phi=\psi$, and $g \in C(T, E)$ can be written as $g=h_{1} g+h_{2} g$, we have $\Phi=\psi=0$. But, this contradicts $\|\Phi\|=\|\Psi\|=1$. Therefore, we must have that either $\sup _{t \varepsilon T \sim K^{\circ}}\|f(t)\|<1$, or there exists $t_{0} \varepsilon T \sim K^{\circ}$ with $\left\|f\left(t_{0}\right)\right\|=1$, for any compact set $K \subseteq T$.

(III) Finally we show that (i), (i), ( since $\|f\|=1$, we see that there exists a $t_{0} \varepsilon T$ with $\left\|f\left(t_{0}\right)\right\|=1$; and from (I), $\left\|f\left(t_{0}\right)\right\|>\|f(t)\|$ for all $t \neq t_{0}$. Again by (II), if $K \subseteq T$ is a compact set with $t_{0} \varepsilon K^{\circ}$, then $\sup _{t \in T \sim K}\|f(t)\|<1$. If there exist distinct functionals $\phi_{1}, \dot{\phi}_{2} \varepsilon E^{*}$ with $\left\|\phi_{1}\right\|=\left\|\phi_{2}\right\|=1$ such that $\phi_{1}\left(f\left(t_{0}\right)\right)=\phi_{2}\left(f\left(t_{0}\right)\right)=1$, then this implies that $\delta_{\phi_{1}, t_{0}}, \delta_{\phi_{2}}, t_{0} \varepsilon C(T, E) *$ are distinct support functionals to the ball in $C(T, E)$ at $f$, which contradicts the fact that $f$ is a smooth point. Therefore $f\left(t_{0}\right)$ is a smooth point of $E$. 
B.. Conversely, suppose that $f \in C(T, E),\|f\|=1$, and (i $),(\underline{i i})$, and (iij) hold; then there exists a unique $t_{0} \varepsilon T$ such that $\left\|f\left(t_{0}\right)\right\|=1$, there exists a compact set $K \subseteq T$ with $t_{0} \varepsilon K^{\circ}$ such that $\sup _{t_{\varepsilon} T \sim K}\|f(t)\|<1$, and $E$ is smooth at $f\left(t_{0}\right)$.

Let $g \in C(T, E), g \neq 0$, and let $\delta>0$ be such that $\|f(t)\|<\left\|f\left(t_{0}\right)\right\|-\delta$ for all $t \varepsilon T \sim K$. If $0<|\lambda|<\frac{\delta}{2\|g\|}$, then for $t \varepsilon T \cdot K$ we have $\|f(t)+\lambda g(t)\| \leq\|f(t)\|+|\lambda| \quad\|g(t)\|<\left\|f\left(t_{0}\right)\right\|+|\lambda|\|g\|-\delta<\left\|f\left(t_{0}\right)\right\|-\frac{\delta}{2}$.

Thus, $\|f(t)+\lambda g(t)\|<\left\|f\left(t_{0}\right)\right\|-\frac{\delta}{2}$ for all $t \in T \sim K$ whenever $0<|\lambda|<\frac{\delta}{2\|g\|}$ On the other hand, $\|f+\lambda g\| \geq\|f\|-|\lambda|\|g\|=\left\|f\left(t_{0}\right)\right\|-|\lambda|\|g\|>\left\|f\left(t_{0}\right)\right\|-\frac{\delta}{2} \quad$ for $0<|\lambda|<\frac{\delta}{2\|g\|}$. Therefore, for $0<|\lambda|<\frac{\delta}{2\|g\|}$,

$$
\sup _{t \varepsilon T}\|f(t)+\lambda g(t)\|=\sup _{t_{\varepsilon} K}\|f(t)+\lambda g(t)\| .
$$

Since $K$ is compact, by Kondagunta's result [6],

$$
\lim _{\lambda \rightarrow 0} \frac{\sup _{t \varepsilon K}\|f(t)+\lambda g(t)\|-\sup _{t \varepsilon K}\|f(t)\|}{\lambda}=\lim _{\lambda \rightarrow 0} \frac{\|f \pm \lambda g\|-\|f\|}{\lambda}
$$

exists for all $g \in C(T, E)$. Hence $C(T, E)$ is smooth at $f$.

q.e.d.

An analogous result holds for the Fréchet differentiability of the norm in $C(T, E)$.

COROLLARY 3.2. Let $T$ be a locally compact Hausdorff space and $E$ a Banach space. The norm function $\|\|:. C(T, E) \rightarrow \mathbb{R}^{+}$is Fréchet differentiable at $f \in C(T, E), \quad f \neq 0$, if and only if

(i) there exists a unique $t_{0} \varepsilon T$ such that $\left\|f\left(t_{0}\right)\right\|>\sup _{t \neq t_{0}}\|f(t)\|$;

(ii) $\left\{t_{0}\right\}$ is an open subset of $T$, that is, $t_{0}$ is an isolated point of $T$;

(iii) the norm function $\|\cdot\|: E \rightarrow \mathbb{R}^{+}$is Fréchet differentiable at $f\left(t_{0}\right)$. (Note: (ii) follows from (i).) 
PROOF.

A. Suppose that $\|\cdot\|: C(T, E) \rightarrow \mathbb{R}^{+}$is Fréchet differentiable at $f \varepsilon C(T, E),\|f\|=1$; then the ball in $C(T, E)$ is smooth at $f$, so there exists a $t_{0} \varepsilon T$ and a compact neighborhood $k$ of $t_{0}$ such that

(1) $\left\|f\left(t_{0}\right)\right\|>\|f(t)\|$ for all $t \varepsilon T, t \neq t_{0}$,

(2) $\sup _{t \in T \sim K}\|f(t)\|<1$,

(3) $E$ is smooth at $f\left(t_{0}\right)$.

Now

$$
\lim _{\lambda \rightarrow 0} \frac{\|f+\lambda g\|-\|f\|}{\lambda}=\lim _{\lambda \rightarrow 0} \frac{\sup _{\varepsilon K}\|f(t)+\lambda g(t)\|-\sup _{t \varepsilon K}\|f(t)\|}{\lambda}
$$

exists uniformly for $g \in B_{C} C(T, E)$, and since $K$ is compact, an appeal to the result of Cox and Nadler [3] shows that $\left\{t_{0}\right\}$ is open and $\|\cdot\|: E \rightarrow \mathbb{R}^{+}$is Fréchet differentiable at $f\left(t_{0}\right)$. Also, since $\left\{t_{0}\right\}$ is open and $K \sim\left\{t_{0}\right\}$ is compact, the uniqueness of $t_{0}$ shows that $\sup _{t \neq t_{0}}\|f(t)\|<1$.

B. Conversely, suppose that (i), (ii), and (iii) hold. Using the previous theorem, we see that $\|\cdot\|: C(T, E) \rightarrow \mathbb{R}^{+}$is Gateaux differentiable at $f$, and taking $K=\left\{t_{0}\right\}$,

$$
\lim _{\lambda \rightarrow 0} \frac{\|f+\lambda g\|-\|f\|}{\lambda}=\lim _{\lambda \rightarrow 0} \frac{\left\|f\left(t_{0}\right)+\lambda g\left(t_{0}\right)\right\|-\left\|f\left(t_{0}\right)\right\|}{\lambda}
$$

exists uniformly for $g \in B_{C}(T, E)$ since $\|\cdot\|: E \rightarrow \mathbb{R}^{+}$is Fréchet differentiable at $f\left(t_{0}\right)$.

4. APPLICATIONS.

A. $\ell_{\infty}(E)$. If $E$ is a Banach space, then

$$
\ell_{\infty}(E)=\left\{x=\left\{x_{n}\right\}_{n \geq 1} \mid \quad x_{n} \in E \text { for } n \geq 1 \text { and } \sup _{n \geq 1}\left\|x_{n}\right\|<\infty\right\}
$$

with the supremum norm, $\|x\|=\sup _{n \geq 1}\left\|x_{n}\right\|$. 
THEOREM 4.1. Let $E$ be a Banach space, then the norm function $\|\cdot\|: \ell_{\infty}(E) \rightarrow \mathbb{R}^{+}$is Gateaux (Fréchet) differentiable at $x=\left\{x_{n}\right\}_{n \geq 1}, \quad x \neq 0$, if and only if

(i) there exists an $n_{0}$ such that $\left\|x_{n_{0}}\right\|>\left\|x_{n}\right\|$ for $n \neq n_{0}$,

(ii) $\sup _{n \neq n_{0}}\left\|x_{n}\right\|<\|x\|$,

(iii) the norm function $\|\cdot\|: E \rightarrow \mathbb{R}^{+}$is Gateaux (Fréchet) differentiable at $x_{n_{0}}$.

PROOF. Let $\mathbb{N}$ denote the set of positive integers equipped with the discrete topology, then $\ell_{\infty}(E)=C(\mathbb{N}, E)$ the space of bounded continuous $E$-valued functions on the locally compact Hausdorff space $N$.

q.e.d.

B. $B\left(\ell_{1}, E\right)$. Let $E$ be a Banach space, let $\ell_{1}$ be the Banach space of all absolutely summable real valued sequences with $\|a\|=\sum_{n=1}^{\infty}\left|a_{n}\right|$ for $a=\left\{a_{n}\right\}_{n \geq 1} \varepsilon \ell_{1}$, and let $B\left(\ell_{1}, E\right)$ be the space of all bounded 1 inear operators from $\ell_{1}$ into $E$. For $n \geq 1$, let $\delta^{n}$ be the $n^{\text {th }}$ basis vector in $\ell_{1}$, that is $\delta^{n}=\left\{\delta_{k}^{n}\right\}_{k \geq 1}$.

THEOREM 4.2. Let $E$ be a Banach space, then the norm function $\|\cdot\|: B\left(\ell_{1}, E\right) \rightarrow \mathbb{R}^{+}$is Gateaux (Fréchet) differentiable at $T \varepsilon B\left(\ell_{1}, E\right)$, $T \neq 0$, if and only if

(i) there exists an $n_{0}$ such that $\left\|T\left(\delta^{n_{0}}\right)\right\|>\left\|T\left(\delta^{n}\right)\right\|$ for $n \neq n_{0}$;

(ii) $\sup _{n \neq n_{0}}\left\|T\left(\delta^{n}\right)\right\|<\|T\|$;

(iii) the norm function $\|\|:. E \rightarrow \mathbb{R}^{+}$is Gateaux (Fréchet) differentiable at $T\left(\delta^{n_{0}}\right)$.

PROOF. The mapping $\sigma: B\left(\ell_{1}, E\right) \rightarrow \ell_{\infty}(E)$ given by $\sigma(T)=\left\{T\left(\delta^{n}\right)\right\}_{n \geq 1}$ for $T \in B\left(\ell_{1}, E\right)$, is a linear isometry of $B\left(\ell_{\uparrow}, E\right)$ onto $\ell_{\infty}(E)$. REMARKS.

1. In connection with the second example, it should be mentioned that Kheinrikh [5] has given a complete characterization of the points of Gateaux and Fréchet 
differentiability of the norm in $K(E, F)$, the space of compact linear operators from $E$ into $F$, where $E$ and $F$ are Banach spaces. He has also given a characterization of the points of Fréchet differentiability of the norm in $B(E, F)$, the space of bounded linear operators from $E$ into $F$ (no proofs are given in this paper). However, the more difficult question of smoothness in $B(E, F)$ is still unanswered.

2. Regarding Theorem 3.1. perhaps this will clear up the popular misconception that, for $T$ locally compact Hausdorff, $C(T, E)$ (or $C(T, \mathbb{R})$ ) is smooth at $f$ if and only if $f$ peaks at some $t_{0} \varepsilon T$. (See e.g. Holmes [4], p. 232, \#4.10). A result which is obviously false, as the example in the intrcduction demonstrates.

\section{REFERENCES}

1. BANACH, S. Théorie des Opérations Linéaires, Monografie Mat. 1, Warsaw, 1932.

2. CECH, E. "On bicompact spaces" Ann. of Math. 38, 823-844, 1937.

3. COX, S.H. Jr. and S.B. NADLER, Jr. "Supremum norm differentiability" Ann. Soc. Math. Polonae 15, 127-131, 1971.

4. HOLMES, R.B. Geometric Functional Analysis and its Applications, Graduate Texts in Mathematics 24, Springer-Verlag, New York, 1975.

5. KHEINRIKH, S. "The differentiability of the norm in spaces of operators" Funkcional Anal. i Prilozen 9 (4), 93-94, 1975. English translation: Functional Anal. Appl. 9 (4), 360-362, 1975.

6. KONDAGUNTA, W. "Some geometric properties of the unit cell in the space $C(X, B) "$ Bull. Acad. Polon. Sci., Ser. Math. Astron. Phys. 19 (11), 1007-1012, 1971.

7. KÖTHE, G. Topologische linear Raume I, Die grundlehren der math. Wissenschaften 107, Springer-Verlag, Berlin, 1966. English translation: Die grundlehren der math. Wissenschaften 159, Springer-Verlag, New York, 1969.

8. MAZUR, S. "Über konvexe Mengen in linearen normierten Răumen" Studia Math. 4, 70-84, 1933. 


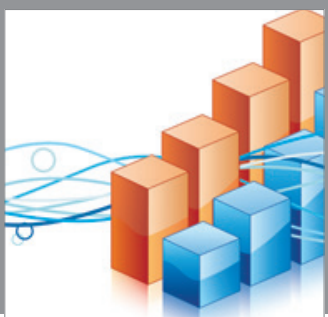

Advances in

Operations Research

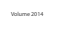

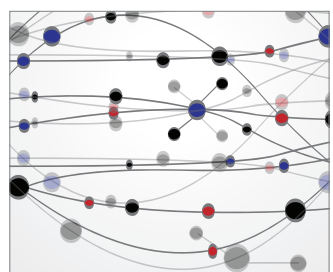

\section{The Scientific} World Journal
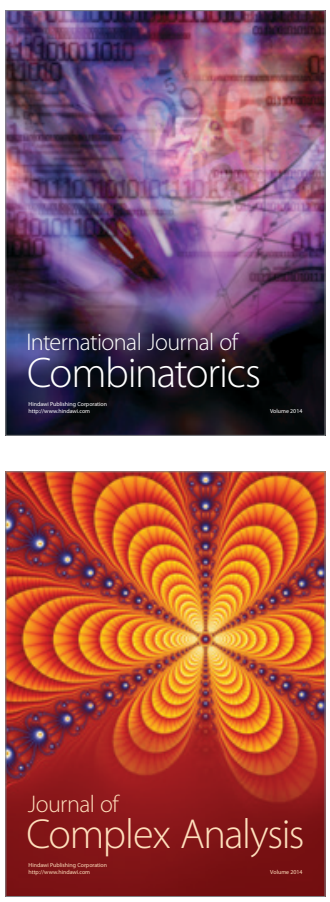

International Journal of

Mathematics and

Mathematical

Sciences
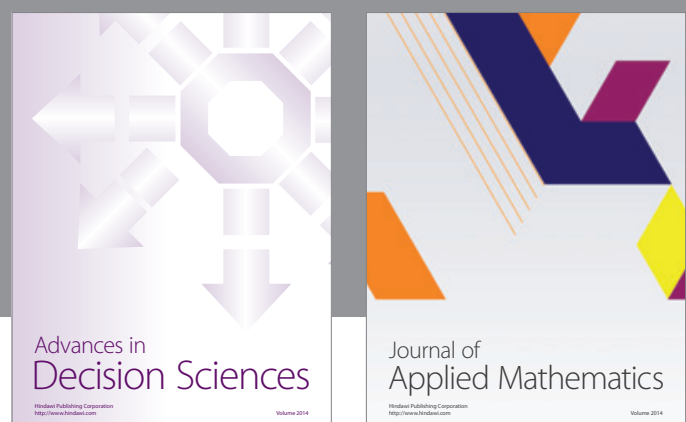

Journal of

Applied Mathematics
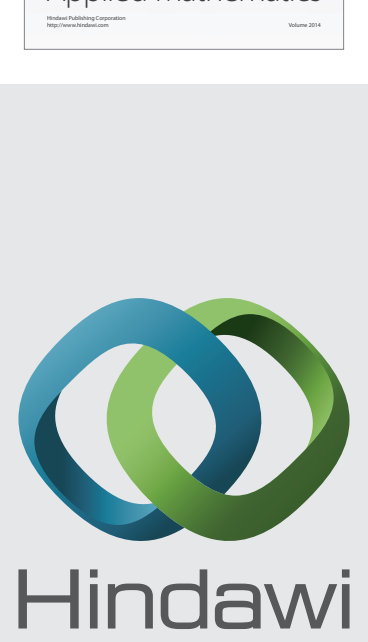

Submit your manuscripts at http://www.hindawi.com
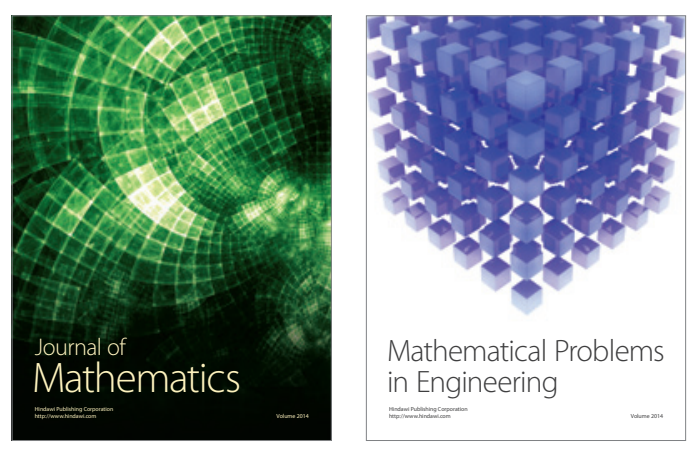

Mathematical Problems in Engineering
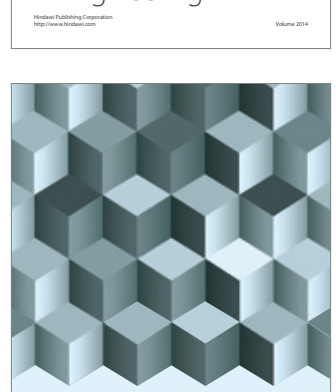

Journal of

Function Spaces
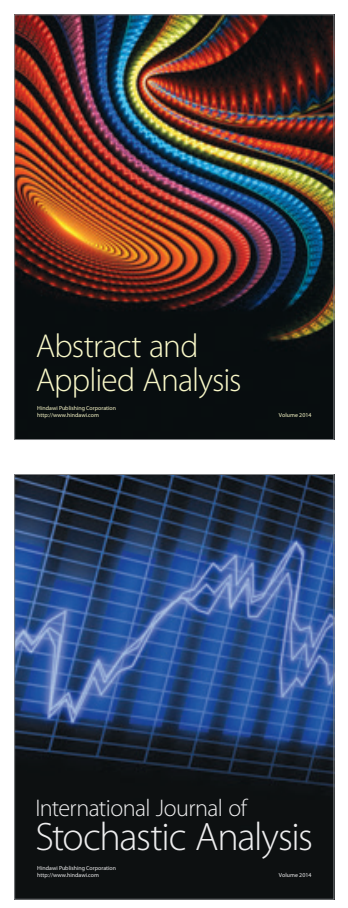

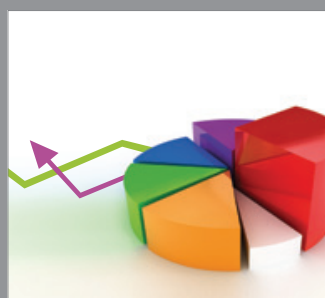

ournal of

Probability and Statistics

Promensencen
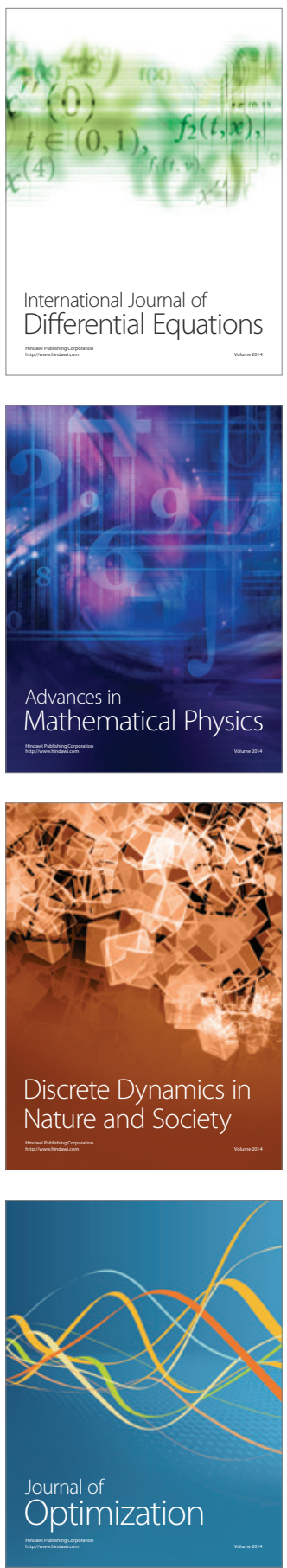